\title{
Family support and vital exhaustion in patients with chronic obstructive pulmonary disease
}

\author{
Athanasios Tselebis ${ }^{1 *}$, Dionisios Bratis ${ }^{1}$, Georgios Moussas $^{1}$, Athanasios Karkanias $^{1}$, Silvia Dumitru ${ }^{2}$, \\ Paraskevi Kavoura², Sofia Lagou², Epaminondas Kosmas², Nikolaos Tzanakis ${ }^{3}$, Nikolaos Siafakas ${ }^{3}$ \\ From $1^{\text {st }}$ International Congress on Neurobiology and Clinical Psychopharmacology \\ and European Psychiatric Association Conference on Treatment Guidance \\ Thessaloniki, Greece. 19-22 November 2009
}

\section{Background}

Many studies have attempted to delineate the relationship between the input in family support (FS) of patients with acute or chronic disease, as well as the effect of this support in the confrontation of illness. These studies showed a negative cross-correlation between the sense of family support and depressive or anxiety symptoms [1,2]. On the other hand, it has been observed that the presence of vital exhaustion (VE), characterized by unusual tiredness, is an aggravating factor, especially in patients with cardiovascular diseases [3,4]. Aim of the study is to investigate the association between the sense of family support and the degree of vital exhaustion in patients with chronic obstructive pulmonary disease (COPD).

\section{Materials and methods}

One hundred and four (87 males and 17 females) outpatients with COPD participated in the study. Family support and vital exhaustion were assessed by using the 13-item Julkunen Family Support Scale (FSS) and the Maastricht Questionnaire (MQ), respectively. Age and education level were also recorded.

\section{Results}

Mean age was $65.3( \pm 8.1)$ and mean education level was 10.97 ( \pm 4.2 , in years). As to clinical measurements, mean FSS score was $54.87( \pm 7.1)$, whereas mean MQ score was $19.83( \pm 8.46)$, which is significant higher than the corresponding score (14.94) of the general population (sample $\mathrm{t}$ - test $\mathrm{p}<0.01$ ). No correlation was observed between age, education level, FS and VE
(Pearson correlation $\mathrm{p}>0.05$ ). In contrary, a strong negative correlation was presented between FS and VE (Pearson correlation $\mathrm{p}<0.05$ )

\section{Conclusions}

Vital exhaustion seems to be present also in patients with COPD. However, further studies are required in order to clarify its associations with the comorbidities of depression and anxiety, which are common in these patients. Finally, our findings suggest the protective role of the sense of family support against vital exhaustion.

\section{Author details}

${ }^{1}$ Psychiatric Department, Sotiria General Hospital of Chest Diseases, Athens, Greece. ${ }^{2}$ Pulmonary Rehabilitation Centre, Sotiria General Hospital of Chest Diseases, Athens, Greece. ${ }^{3}$ Department of Thoracic Medicine, University of Crete Medical School, Heraklion, Greece.

Published: 22 April 2010

\section{References}

1. Bratis D, Tselebis A, Sikaras C, Moulou A, Giotakis K, Zoumakis E, llias I: Alexithymia and its association with burnout, depression and family support among Greek nursing staff. Hum Resour Health 2009, 7:72.

2. Ilias I, Tselebis A, Theotoka I, Hatzimichelakis E: Association of perceived family support through glycemic control in native Greek patients managing diabetes with diet alone. Ethn Dis 2004, 14(1):2.

3. Appels A, Höppener P, Mulder P: A questionnaire to assess premonitory symptoms of myocardial infarction. International journal of cardiology 17:15-24.

4. Anagnostopoulou T, Kioseoglou G: The Greek adaptation of the Maastricht Questionnaire for the assessment of vital exhaustion. Scientific Yearbook of the Psychology Department Aristotle University of Thessaloniki, GreeceSygkolitou E, Gonida-Bamniou E 3:137-152, (in Greek).

doi:10.1186/1744-859X-9-S1-S161

Cite this article as: Tselebis et al.: Family support and vital exhaustion in patients with chronic obstructive pulmonary disease. Annals of General Psychiatry 2010 9(Suppl 1):S161 